Journal of

Applied

Crystallography

ISSN 1600-5767

\title{
Osmotic shrinkage of sterically stabilized liposomes as revealed by time-resolved small-angle X-ray scattering
}

\author{
Zoltán Varga, András Wacha and Attila Bóta
}

J. Appl. Cryst. (2014). 47, 35-40

Copyright (C) International Union of Crystallography

Author(s) of this paper may load this reprint on their own web site or institutional repository provided that this cover page is retained. Republication of this article or its storage in electronic databases other than as specified above is not permitted without prior permission in writing from the IUCr.

For further information see http://journals.iucr.org/services/authorrights.html

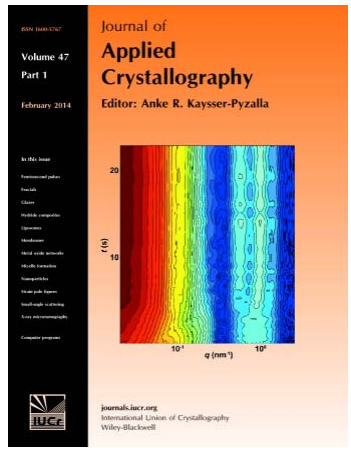

Many research topics in condensed matter research, materials science and the life sciences make use of crystallographic methods to study crystalline and non-crystalline matter with neutrons, X-rays and electrons. Articles published in the Journal of Applied Crystallography focus on these methods and their use in identifying structural and diffusioncontrolled phase transformations, structure-property relationships, structural changes of defects, interfaces and surfaces, etc. Developments of instrumentation and crystallographic apparatus, theory and interpretation, numerical analysis and other related subjects are also covered. The journal is the primary place where crystallographic computer program information is published.

Crystallography Journals Online is available from journals.iucr.org 
Journal of

Applied

Crystallography

ISSN 1600-5767

Received 17 May 2013

Accepted 7 November 2013

(C) 2014 International Union of Crystallography

\section{Osmotic shrinkage of sterically stabilized liposomes as revealed by time-resolved small-angle $\mathrm{X}$-ray scattering 1}

\author{
Zoltán Varga, ${ }^{*}$ András Wacha and Attila Bóta \\ Department of Biological Nanochemistry, Institute of Molecular Pharmacology, Research Centre for \\ Natural Sciences, Hungarian Academy of Sciences, Pusztaszeri út 59-67, H-1025, Budapest, \\ Hungary. Correspondence e-mail: varga.zoltan@ttk.mta.hu
}

\begin{abstract}
Time-resolved synchrotron small-angle X-ray scattering (SAXS) was used to study the structural changes during the osmotic shrinkage of a pharmacologically relevant liposomal drug delivery system. Sterically stabilized liposomes (SSLs) with a diameter of $100 \mathrm{~nm}$ and composed of hydrogenated soy phosphocholine, cholesterol and distearoyl-phosphoethanolamine-PEG 2000 prepared in a salt-free buffer were mixed with a buffered $0.3 \mathrm{M} \mathrm{NaCl}$ solution using a stopped flow apparatus. The changes in the liposome size and the bilayer structure were followed by using SAXS with a time resolution of $20 \mathrm{~ms}$. A linear decrease in liposome size is observed during the first $\sim 4 \mathrm{~s}$ of the osmotic shrinkage, which reveals a water permeability value of $0.215(15) \mu \mathrm{m} \mathrm{s}^{-1}$. The change in the size of the liposomes upon the osmotic shrinkage is also confirmed by dynamic light scattering. After this initial step, broad correlation peaks appear on the SAXS curves in the $q$ range of the bilayer form factor, which indicates the formation of bi- or oligolamellar structures. Freeze-fracture combined with transmission electron microscopy revealed that lens-shaped liposomes are formed during the shrinkage, which account for the appearance of the quasi-Bragg peaks superimposed on the bilayer form factor. On the basis of these observations, it is proposed that the osmotic shrinkage of SSLs is a twostep process: in the initial step, the liposome shrinks in size, while the area/lipid adapts to the decreased surface area, which is then followed by the deformation of the spherical liposomes into lens-shaped vesicles.
\end{abstract}

\section{Introduction}

The formation of liposomes from phospholipids in excess water was first observed in the early 1960s by Alec D. Bangham (Bangham \& Horne, 1964; Bangham, 1993), and since this discovery the use of liposomes as models for cell membranes as well as their application as drug delivery vehicles has become widespread (Edidin, 2003; Chang \& Yeh, 2012). The clinical application of liposomal drugs was hampered by the fast clearing of conventional liposomes from the blood stream, until the appearance of 'stealth' or sterically stabilized liposomes (SSLs), in which a longer in vivo circulation time is assured by the incorporation of polyethylene glycol (PEG)-containing phospholipids within the liposomal constituents (Allen et al., 1995; Papahadjopoulos et al., 1991; Woodle, 1995).

Physicochemical characterization of SSLs is not only important from the side of basic understanding of the structure-function relationship of liposomal drugs; it has also been

\footnotetext{
$\mathbf{1}$ This article will form part of a virtual special issue of the journal, presenting some highlights of the 15th International Small-Angle Scattering Conference (SAS2012). This special issue will be available in early 2014.
}

requested by the regulatory bodies for new liposomal products (Jiang et al., 2011; Barenholz, 2012). A wide variety of methods ranging from spectroscopic methods to morphological characterization have been utilized to study liposomes (Jiang et al., 2011). Among these, small-angle X-ray scattering (SAXS) proved to be an excellent method to describe relevant properties of liposomes, such as lamellarity, bilayer thickness, area/ lipid and the thickness of the PEG layer (Pabst et al., 2010; Bouwstra et al., 1993; Brzustowicz \& Brunger, 2005; Varga et al., 2010, 2012).

Water permeability is one of the basic physicochemical properties of SSLs, which might be the reason for the lack of studies in this topic. On the other hand, the permeability of water and other small molecules through the lipid bilayer has been extensively studied for more than 40 years (Reeves \& Dowben, 1970; Haran \& Shporer, 1976; Boroske et al., 1981; Huster et al., 1997; Olbrich et al., 2000; Mathai et al., 2008; Nagle et al., 2008). Many important aspects of diffusion of water through the phospholipid bilayer, like the dependence of permeability on the area/lipid of the bilayer, have been revealed, but there are still unanswered questions in this field. The difficulty of measuring the absolute value of membrane 
water permeability is an important factor in this regard. Differences up to one order of magnitude can be seen between measurements by different experimental techniques (Haines \& Liebovitch, 1995), which originate presumably from the fact that most of the methods employed use a tracer molecule or ion. The commonly used measurement of volume change of liposomes is based on the self-quenching of an entrapped fluorophore (e.g. carboxyfluorescein), while NMR measurements utilize a paramagnetic ion or its complex (Lande et al., 1995; Haran \& Shporer, 1976; Huster et al., 1997). The method of phase contrast microscopy using giant unilamellar vesicles is a label-free technique (Boroske et al., 1981), but as a nonensemble method it gives information about the individual vesicles and cannot be used for liposomes with a size of around $100 \mathrm{~nm}$. Turbidity measurement combined with a stopped-flow apparatus was also applied for the measurement of water permeability but only reported for liposomes in the size range of 0.5-10 $\mu \mathrm{m}$ (Reeves \& Dowben, 1970).

In this article, the use of time-resolved SAXS to follow the changes in the liposome size during osmotic shrinkage is presented, which provides a description of the water permeability of liposomes of $100 \mathrm{~nm}$ in size without the need of any additive tracer molecule. Additionally, the applied method enables the parallel characterization of the bilayer structure, giving new insights into the process of osmotic shrinkage of SSLs.

\section{Experimental}

\subsection{Materials and methods}

Synthetic high-purity hydrogenated soy lecithin (HSPC) and 1,2-distearoyl-sn-glycero-3-phosphoethanolamine- $N$-[methoxy (polyethylene glycol)-2000] (DSPE-PEG 2000) were purchased from NOF Corporation (Japan) and Avanti Polar Lipids (USA), respectively. Cholesterol was purchased from Sigma-Aldrich (Hungary). All of the chemicals were used without further purification. SSL samples were prepared by the hydration, freeze-thaw and extrusion method. Briefly, the components in the weight ratios of HSPC:DSPE-PEG 2000: cholesterol $=3: 1: 1$ (corresponding to the molar ratios of 0.565:0.053:0.382) were dissolved in a chloroform:methanol mixture $(2: 1 \mathrm{v} / \mathrm{v})$. The composition used is identical to that of the first commercially available liposomal drug, namely Doxil/ Caelyx (US/EU). The solvent was then evaporated at $313 \mathrm{~K}$ and the resulting lipid film was kept under vacuum overnight to remove residual traces of the solvent. A $10 \mathrm{~m} M$ Tris $\mathrm{pH} 7.4$ buffer solution (Sigma-Aldrich, Hungary) made with ultrapure water $(18.2 \mathrm{M} \Omega \mathrm{cm})$ was added to the sample to obtain a total lipid concentration of $16 \mathrm{mg} \mathrm{ml}^{-1}$. Ten freeze-thaw cycles using liquid nitrogen and a lukewarm water bath were applied for homogenization. Finally, the samples were extruded ten times through $100 \mathrm{~nm}$ pore size polycarbonate filters (Nucleopore, Whatman Inc.) using a LIPEX extruder (Northern Lipids Inc., Canada). The extrusion was performed at $333 \mathrm{~K}$.

\subsection{SAXS measurements}

Time-resolved SAXS measurements were carried out at the ID02 beamline (Narayanan et al., 2001) at ESRF (Grenoble, France) using a stopped-flow rapid mixing device (BioLogic, SFM-400, Grenoble, France). The energy of the incoming $\mathrm{X}$-ray beam was set to $12 \mathrm{keV}$, and the two-dimensional scattering patterns were collected with a FReLoN 2000 CCD detector. After correcting for geometrical distortions and normalizing to primary-beam intensity and sample transmission, the scattering curves were obtained by radial averaging of the two-dimensional scattering patterns. Finally, the calibration of the curves to absolute units of macroscopic cross section $\left(\mathrm{cm}^{-1}\right)$ was performed by using the scattering of water as a reference.

Osmotic shrinkage of liposomes was initiated by mixing the liposome sample prepared in a salt-free buffer $(10 \mathrm{~m} M$ Tris $\mathrm{pH}$ 7.4) with 0.3 $M \mathrm{NaCl}$ solution in the same buffer using 1:1 volume ratio. The first SAXS measurement was performed at $20 \mathrm{~ms}$ after mixing with an exposure time of $10 \mathrm{~ms}$, while the second measurement was made after an initial time step of $200 \mathrm{~ms}$. The subsequent exposures were made using the previous time step multiplied by a factor of 1.05. All the experiments were repeated at least three times. In order to rule out possible artefacts arising from effects other than the osmotic pressure, mixing of the liposome sample with a saltfree buffer was also carried out.

\subsection{Dynamic light scattering (DLS)}

DLS measurements were performed on a W130i apparatus (Avid Nano Ltd, High Wycombe, UK) and using a low-volume disposable cuvette (UVette, Eppendorf Austria GmbH). First, $70 \mu \mathrm{l}$ of the liposome sample prepared in a salt-free buffer was measured ten times for $30 \mathrm{~s}$. This was followed by addition of $70 \mu \mathrm{l}$ of buffered saline solution (10 $\mathrm{m} M$ Tris $\mathrm{pH} 7.4,0.3 \mathrm{M}$ $\mathrm{NaCl}$ ), and the sequence with $30 \mathrm{~s}$ measurements with ten repeats was started again. In a control measurement, the liposome sample was mixed with a salt-free buffer and the same procedure was repeated.

\subsection{Freeze-fracture transmission electron microscopy (FF- TEM)}

After initiating the osmotic shrinkage of the SSL liposomes with the buffered $0.3 \mathrm{M} \mathrm{NaCl}$ solution, $2 \mu \mathrm{l}$ of the sample was pipetted onto a gold sample holder, frozen by plunging it immediately into partially solidified Freon for $20 \mathrm{~s}$ and stored in liquid nitrogen. Fracturing was performed at $173 \mathrm{~K}$ in a Balzers freeze-fracture device (Balzers BAF 400D, Balzers AG, Liechtenstein). Replicas of the fractured faces etched at $173 \mathrm{~K}$ were made by platinum-carbon shadowing and then cleaned with a water solution of surfactant and washed with distilled water. The replicas were placed on 200 mesh copper grids and examined in a MORGAGNI 268D (FEI, The Netherlands) transmission electron microscope. 


\section{Results and discussion}

The SAXS curve of unilamellar liposomes carries information about the overall size and shape of the whole particles as well as about the bilayer structure. Liposomes with nominal diameter of $100 \mathrm{~nm}$ exhibit a Guinier plateau in the $q$ range below $0.05 \mathrm{~nm}^{-1}$, while the width of the size distribution $(\Delta R /$ $R$ ) of the samples prepared by extrusion is around $0.2-0.3$, which results a smeared minima at $q \simeq 4.5 / R$ (i.e. around $q=$ $0.09 \mathrm{~nm}^{-1}$ for $R=50 \mathrm{~nm}$ ).

Upon mixing of a salt-free sterically stabilized liposome sample with $0.3 \mathrm{M} \mathrm{NaCl}$ solution, traceable changes occur in the liposome size and in the bilayer structure within the time range of a few seconds. Fig. 1 shows the scattering curves in every fifth time step elapsed since the mixing, while the full set of curves for the whole time interval is shown in Fig. 2 as a colour-coded intensity map. Fig. 3 shows the Guinier plot of the initial parts of the curves shown in Fig. 1, which confirm

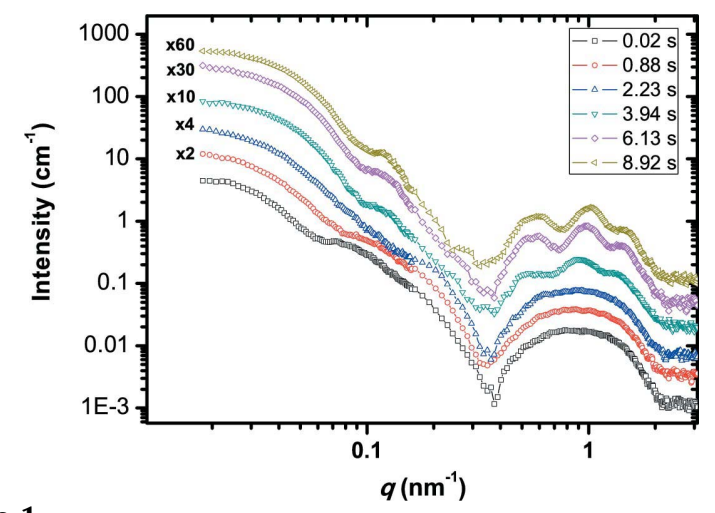

Figure 1

The time evolution of the SAXS curves of the SSL sample after mixing it with $0.3 \mathrm{M} \mathrm{NaCl}$ solution (in 1:1 volume ratio). The curves from every fifth time step are shown shifted vertically by the factors indicated at their origin for better visualization.

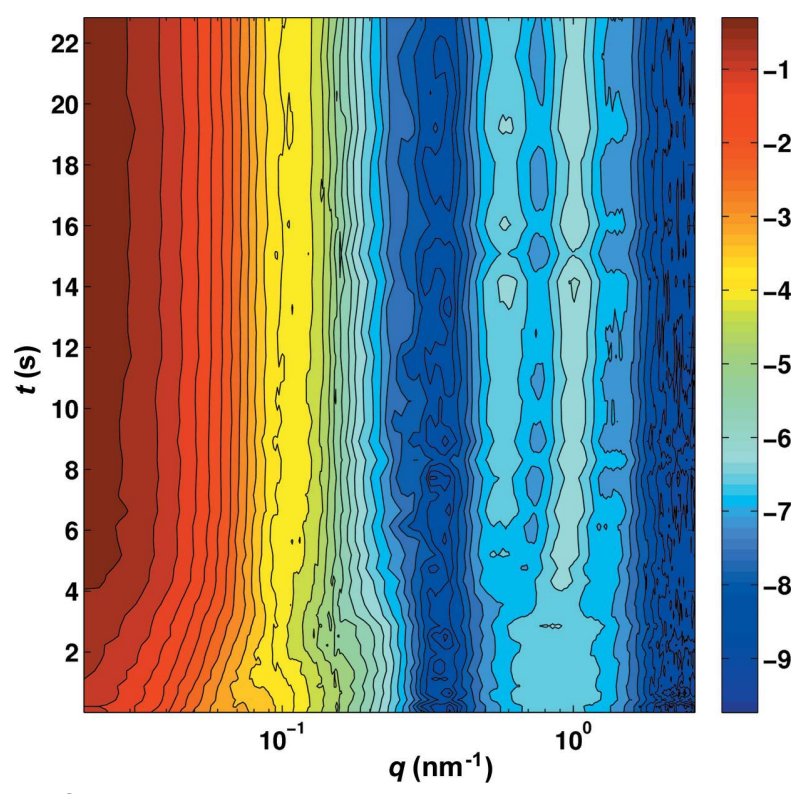

Figure 2

Colour-coded intensity map of the time-resolved SAXS experiment for the whole time interval. the presence of monodisperse liposomes and the fact that the size of the liposomes shrinks within $\sim 4 \mathrm{~s}$ after mixing. DLS measurements were also performed in order to confirm the decrease in the size of the liposomes during osmotic shrinkage. Fig. 4 shows the change in the hydrodynamic diameter of the liposomes after the addition of the salt solution. According to the DLS results, the initial average hydrodynamic diameter of the liposomes was $117 \mathrm{~nm}$, and after mixing with the salt solution it was $110.8 \mathrm{~nm}$, while no change was observed for the mixing of the liposome sample with buffer solution.

The time evaluation of the radius of gyration obtained from the SAXS curves after the osmotic shock is shown in Fig. 5. In a first approximation, we will use the radius of gyration to refer to the size of the liposomes. According to Fig. 5, the shrinkage of liposomes proceeds linearly starting from $R_{0} \simeq$ $48 \mathrm{~nm}$ until $R_{\text {final }} \simeq 38 \mathrm{~nm}$ within 4 s. Similar linearity was observed for the shrinkage of giant unilamellar egg-lecithin vesicles (Boroske et al., 1981), which is in connection with the water permeability of the membrane. In general, the osmotic flow of water molecules through the bilayer can be written as $j=-P \Delta c$, where $j$ is the molar flux density, $\Delta c$ is the difference of molar concentration between the solutions on

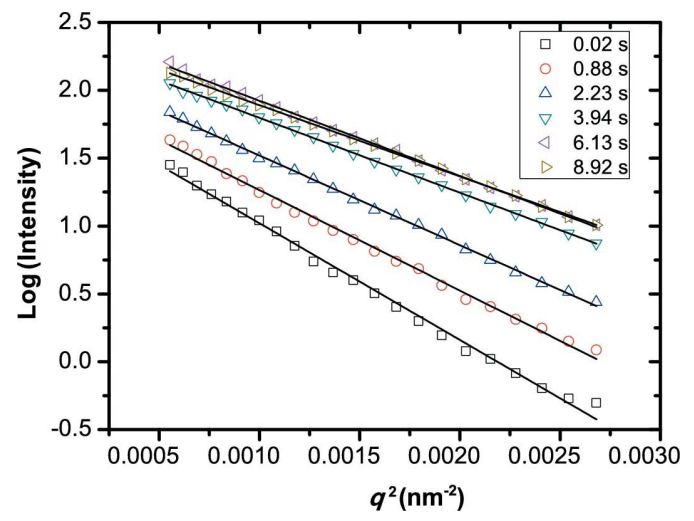

Figure 3

The Guinier plot of the beginning part of the curves shown in Fig. 1.

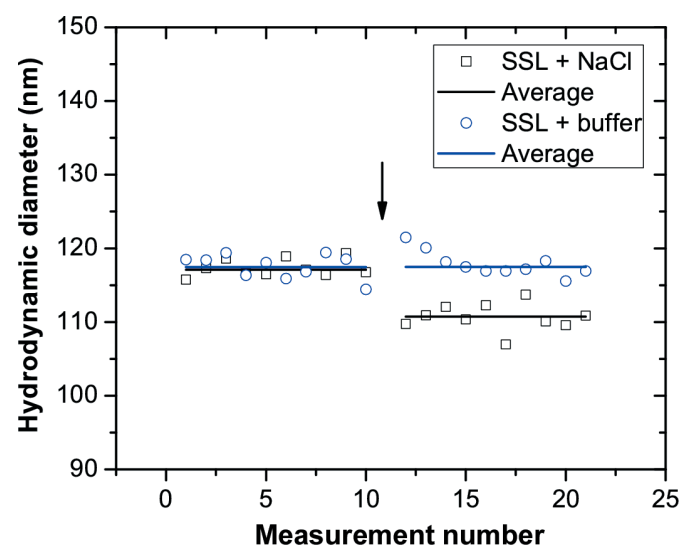

Figure 4

DLS measurements on the osmotic shrinkage of sterically stabilized liposomes. The size of the liposome sample prepared in a salt-free buffer was measured ten times, which was then followed by the addition of buffered saline solution $(0.3 \mathrm{M} \mathrm{NaCl})$, and the measurement sequence was repeated. For the control measurement, the liposome sample was mixed with a salt-free buffer. 
the two sides of the membrane and $P$ is the permeability. The time dependence of the radius of a spherical vesicle upon osmotic shrinkage can be written as

$$
\mathrm{d} R / \mathrm{d} t=-\alpha P \Delta c,
$$

where $\alpha$ is the molar volume of water. The form of equation (1) implies a linear decrease in size in agreement with our observation. A linear fit to the data in the beginning part of the time evolution of the radius of liposomes during osmotic shrinkage results in a slope of $-2.30(17) \mathrm{nm}^{-1}$, which corresponds to a permeability of $0.215(15) \mu \mathrm{m} \mathrm{s}^{-1}$. This is smaller than the reported values for egg-lecithin vesicles (30$40 \mathrm{~m} \mathrm{~s}^{-1}$; Boroske et al., 1981) and liposomes composed of various synthetic phospholipids (2.1 and $15.8 \mu \mathrm{m} \mathrm{s}^{-1}$ for 1,2diauroyl-sn-glycero-3-phosphoethanolamine and 1,2-dioleoylsn-glycero-3-phosphocholine, respectively; Mathai et al., 2008) and phospholipid/cholesterol mixtures (14.5 and $6.8 \mu \mathrm{m} \mathrm{s}^{-1}$ for 1,2-dioleoyl-sn-glycero-3-phosphocholine containing 10 and $40 \%$ cholesterol, respectively; Mathai et al., 2008). In general, the permeability of bilayers composed of phospholipids with saturated fatty acids is smaller than for those containing lipids with unsaturated fatty acids, and cholesterol is also known to reduce water permeability.

The study of osmotic water permeability of PEG-lipidcontaining bilayers is rare, and to the best of our knowledge, the permeability of the HSPC/DSPE-PEG 2000/cholesterol (in a weight ratio of $3: 1: 1$ ) liposome system has not been reported in the literature despite its significance as it corresponds to the lipid composition of Doxil/Caelyx. Terreno et al. (2008) reported the water permeability values of DSPE-PEG 2000-containing liposomes measured by the NMR longitudinal water-proton relaxation rate of vesicles encapsulating a clinically approved gadolinium complex. For liposomes composed of 1,2-dipalmitoyl-sn-glycero-3-phosphocholine/ DSPE-PEG 2000 (in the molar ratio of 95:5) a water permeability value of $0.26(6) \mu \mathrm{m} \mathrm{s}^{-1}$ was reported, which agrees with the value obtained from our time-resolved SAXS experiments.

The reason behind the drastically reduced water permeability values for the PEG-lipid-containing bilayers might be

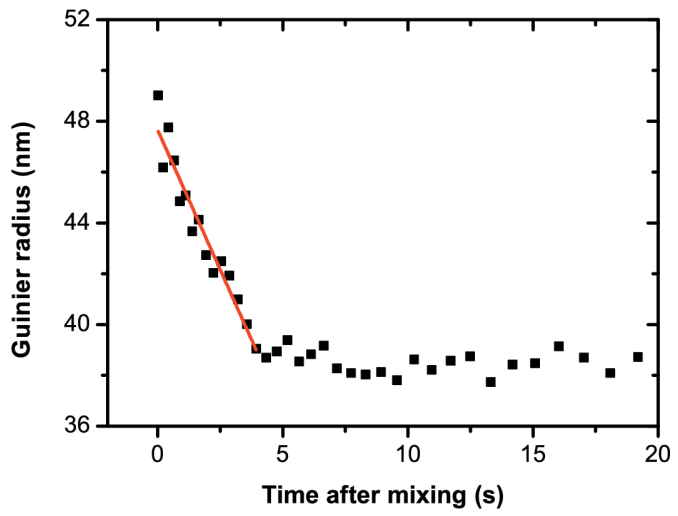

Figure 5

The time evolution of the Guinier radius of the liposomes during the osmotic shrinkage. The first linearly decaying part is used for the determination of the value of the water permeability. that the polymer layer of these sterically stabilized liposomes blocks the area available for water permeation. The latter is in line with the fact that the hydration of the PEG-lipidcontaining bilayers has a minimum at the composition corresponding to $\sim 6 \mathrm{~mol} \%$ of the PEG-lipid, the same as we used in this study (Tirosh et al., 1998; Varga et al., 2013).

Beside the changes in the beginning part of the scattering curves during the osmotic shrinkage, significant alteration can also be detected in the $q$ region of the form factor of the phospholipid bilayer, i.e. at $0.2<q<3 \mathrm{~nm}^{-1}$. This part of the scattering curve carries information about the internal structure of the bilayer, i.e. it can be used to determine the electron density profile along the bilayer normal; hence information on the bilayer thickness and even on the structure of the PEG layer on the surface of the liposomes can be obtained (Bouwstra et al., 1993; Hirai et al., 2003; Brzustowicz \& Brunger, 2005; Varga et al., 2012). Interestingly, there are less pronounced changes in the form factor during the first 2-3 s of the time evolution of the osmotic shrinkage, in the same time interval in which the size of the liposome drops significantly. On the other hand, at the time when the size of the liposome almost reached its final size, broad quasi-Bragg peaks appear superimposed on the bilayer form factor. Such correlation peaks reveal that periodic structures are formed in the liposome system. Three distinct peaks at $q$ values of $\sim 0.5, \sim 1$ and $1.5 \mathrm{~nm}^{-1}$ may correspond to the first, second and third order of a periodic repeat distance of $\sim 12 \mathrm{~nm}$, respectively. The observed $\sim 12 \mathrm{~nm}$ of periodicity is also in line with the lamellar repeat period for distearoylPC/DSPE-PEG 2000 liposomes under osmotic pressure similar to our experiment as reported by Kenworthy et al. (1995). In the case of giant unilamellar vesicles, the formation of daughter vesicles on the inner side of the mother liposome was reported in the literature (Bernard et al., 2002; Ménager \& Cabuil, 2002) and explained as the adoption of a phospholipid bilayer to the decreased surface area caused by the osmotic shrinkage. In our case, since the size range of liposomes is orders of magnitude smaller than in the above cited example, such high local curvature is unlikely to occur. Another explanation for the formation of close bilayer contacts could be the deformation of the spherical liposomes into elliptical or lens-shaped vesicles. The same observation was reported for liposomes composed of dipalmitoyl-phosphocholine/DSPE-PEG 2000 (so-called LIPOCEST) by Terreno et al. (2009). In that study, cryo-TEM was applied to reveal the morphology of the liposomes after the osmotic shrinkage. In order to verify that lens-shaped liposomes are formed during our experiments, we have performed freeze-fracture combined with TEM (FF-TEM) investigations. In the case of the FF-TEM investigations, the sample is frozen very quickly $\left(\sim 10^{5} \mathrm{~K} \mathrm{~s}^{-1}\right)$, which inhibits crystal formation in the aqueous samples to preserve the original morphology. Fig. 6 shows typical FF-TEM images of the osmotically shrunken liposomes. Lens-shaped liposomes are clearly visible on these images, which confirms that the appearance of quasi-Bragg peaks on the SAXS curves can be related to the formation of bilamellar structures upon the shrinkage. A similar observation was made by Deme et al. (2002), who 
studied the lamellar-vesicle unbinding transition in the dioleoylphosphatidylserine (DOPS)/water system using SAXS, SANS and electron microscopy. The analogy between this study and that of Deme et al. (2002) is apparent, as in both cases the transition between a single bilayer phase and a bi- or oligolamellar phase is observed by the appearance of quasi-Bragg peaks superimposed on the bilayer form factor. On the other hand, in our case the ordered phase is a lens-shaped liposome, while in the case of the DOPS/water system it is a multilamellar structure.

According to our time-resolved SAXS experiments, the following schematic scenario can be drawn about the osmotic shrinkage of liposomes: as the osmotic pressure appears on the liposome surface, its size starts to decrease, which is accompanied by water outflow through the bilayer. In the initial steps of the process, the bilayer packing density adapts to the decreased surface. The observed decrease in size $(\Delta R \simeq$ $10 \mathrm{~nm}$ ) implies a change from an initial area/lipid of 0.7 to $0.45 \mathrm{~nm}^{2}$, which cannot be accommodated for a long period of time, and as a result, the spherical liposomes deform into lensshaped ones. The latter results in close contact of the opposite bilayers of the liposomes, which leads to quasi-Bragg peaks on the scattering curves in the $q$ range of the bilayer form factor.
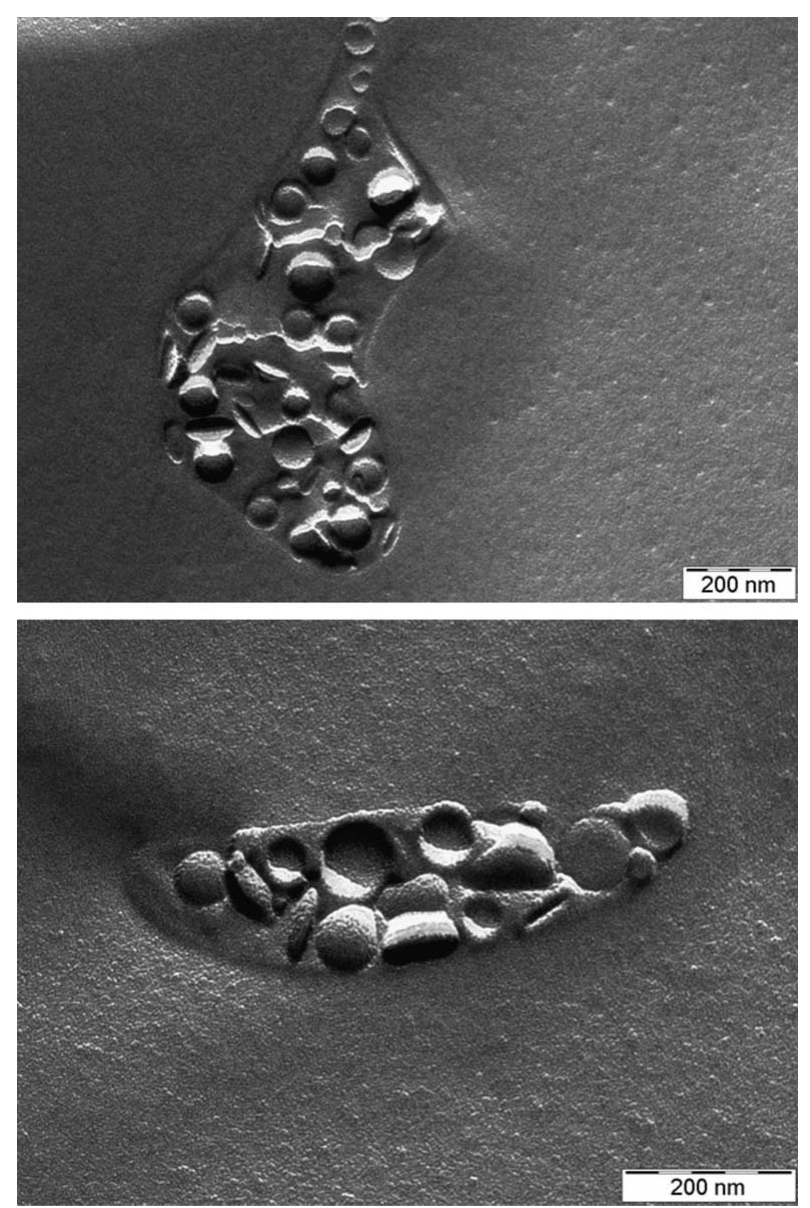

Figure 6

Typical FF-TEM images of the osmotically shrunken liposomes. Lensshaped liposomes with different orientations can be observed.

\section{Conclusion}

Osmotic shrinkage of the pharmacologically relevant HSPC/ DSPE-PEG 2000/cholesterol (in weight ratio of 3:1:1) liposome system has been studied by means of time-resolved SAXS. The liposome sample prepared in a salt-free buffer was mixed with $0.3 \mathrm{M} \mathrm{NaCl}$ solution (in the same buffer) in 1:1 volume ratio using a stopped-flow device and the changes in the scattering curves were monitored by SAXS with a time resolution of $20 \mathrm{~ms}$. A linear decrease in the radius of gyration of the liposomes from $\sim 48$ to $\sim 38 \mathrm{~nm}$ was observed within the first $\sim 4 \mathrm{~s}$ of the experiment, while changes in the bilayer structure appeared 2-3 s after applying the osmotic stress. The change in the overall size of the liposomes was also confirmed by DLS measurements. The water permeability based on the linear part of the time evaluation of the liposome size was found to be $0.215(15) \mu \mathrm{m}^{-1}$, which is in agreement with previous NMR measurements (Terreno et al., 2008) and is significantly lower than the permeability values of pure phospholipid and phospholipid/cholesterol systems (Boroske et al., 1981; Mathai et al., 2008).

It was found that the shrinkage of the SSLs is a two-stage process, i.e. the initial decrease of liposome size is accompanied by a decrease of the area/lipid, which is followed deformation of spherical liposomes into lens-shaped ones. The final morphology of the shrunken liposomes was confirmed by FF-TEM investigations.

The presented method offers a new way of describing the water permeability of a phospholipid bilayer which overcomes the problem of using tracer molecules and ions for the determination of the latter, and as an ensemble method, it gives an average value of the permeability. Moreover, this technique also provides information about structural changes within the bilayer, and it enables the changes to be coupled to the observed decrease in liposome size.

The feasibility of using time-resolved SAXS measurements for the determination of osmotic water permeability is demonstrated on the example of SSLs. The extension of our investigation to liposomes with different compositions and application of sophisticated evaluation techniques to interpret the scattering curves might be the subject of future studies.

This work was supported by the Hungarian Scientific Research Fund (OTKA, Hungary) and the National Innovation Office (NIH, Hungary) under grant agreements CNK81056 and KMR_12-1-2012-0155. We are grateful to the European Synchrotron Radiation Facility for provision of beam time at ID02 (proposal No. SC-3269), and thank J. Gummel and T. Narayanan for their kind help during the measurements.

\section{References}

Allen, T. M., Hansen, C. B. \& de Menezes, D. E. L. (1995). Adv. Drug Deliv. Rev. 16, 267-284.

Bangham, A. D. (1993). Chem. Phys. Lipids, 64, 275-285.

Bangham, A. D. \& Horne, R. W. (1964). J. Mol. Biol. 8, 660-668.

Barenholz, Y. (2012). J. Contrib. Rel. 160, 117-134. 
Bernard, A.-L., Guedeau-Boudeville, M.-A., Jullien, L. \& di Meglio, J.-M. (2002). Biochim. Biophys. Acta Biomembr. 1567, 1-5.

Boroske, E., Elwenspoek, M. \& Helfrich, W. (1981). Biophys. J. 34, 95-109.

Bouwstra, J. A., Gooris, G. S., Bras, W. \& Talsma, H. (1993). Chem. Phys. Lipids, 64, 83-98.

Brzustowicz, M. R. \& Brunger, A. T. (2005). J. Appl. Cryst. 38, 126131.

Chang, H. I. \& Yeh, M. K. (2012). Int. J. Nanomed. 7, 49-60.

Deme, B., Dubois, M., Gulik-Krzywicki, T. \& Zemb, T. (2002). Langmuir, 18, 997-1004.

Edidin, M. (2003). Annu. Rev. Biophys. Biomol. Struct. 32, 257-283.

Haines, T. H. \& Liebovitch, L. S. (1995). Permeability and Stability of Lipid Bilayers, pp. 123-136. Boca Raton: CRC Press.

Haran, N. \& Shporer, M. (1976). Biochim. Biophys. Acta Biomembr. 426, 638-646.

Hirai, M., Iwase, H., Hayakawa, T., Koizumi, M. \& Takahashi, H. (2003). Biophys. J. 85, 1600-1610.

Huster, D., Jin, A. J., Arnold, K. \& Gawrisch, K. (1997). Biophys. J. 73, 855-864.

Jiang, W., Lionberger, R. \& Yu, L. X. (2011). Bioanalysis, 3, 333-344.

Kenworthy, A. K., Hristova, K., Needham, D. \& McIntosh, T. J. (1995). Biophys. J. 68, 1921-1936.

Lande, M. B., Donovan, J. M. \& Zeidel, M. L. (1995). J. Gen. Physiol. 106, 67-84.

Mathai, J. C., Tristram-Nagle, S., Nagle, J. F. \& Zeidel, M. L. (2008). J. Gen. Physiol. 131, 69-76.

Ménager, C. \& Cabuil, V. (2002). J. Phys. Chem. B, 106, 7913-7918.
Nagle, J. F., Mathai, J. C., Zeidel, M. L. \& Tristram-Nagle, S. (2008). J. Gen. Physiol. 131, 77-85.

Narayanan, T., Diat, O. \& Boesecke, P. (2001). Nucl. Instrum. Methods Phys. Res. Sect. A, 467, 1005-1009.

Olbrich, K., Rawicz, W., Needham, D. \& Evans, E. (2000). Biophys. J. 79, 321-327.

Pabst, G., Kucerka, N., Nieh, M. P., Rheinstädter, M. C. \& Katsaras, J. (2010). Chem. Phys. Lipids, 163, 460-479.

Papahadjopoulos, D., Allen, T. M., Gabizon, A., Mayhew, E., Matthay, K., Huang, S. K., Lee, K.-D., Woodle, M. C., Lasic, D. D., Redemann, C. \& Martin, J. F. (1991). Proc. Natl Acad. Sci. USA, 88, 11460-11464.

Reeves, J. P. \& Dowben, R. M. (1970). J. Membr. Biol. 3, 123-141.

Terreno, E., Delli Castelli, D., Violante, E., Sanders, H. M., Sommerdijk, N. A. \& Aime, S. (2009). Chem. Eur. J. 15, 14401448.

Terreno, E., Sanino, A., Carrera, C., Castelli, D. D., Giovenzana, G. B., Lombardi, A., Mazzon, R., Milone, L., Visigalli, M. \& Aime, S. (2008). J. Inorg. Biochem. 102, 1112-1119.

Tirosh, O., Barenholz, Y., Katzhendler, J. \& Priev, A. (1998). Biophys. J. 74, 1371-1379.

Varga, Z., Berényi, S., Szokol, B., Orfi, L., Kéri, G., Peták, I., Hoell, A. \& Bóta, A. (2010). J. Phys. Chem. B, 114, 6850-6854.

Varga, Z., Mihály, J., Berényi, Sz. \& Bóta, A. (2013). Eur. Polym. J. 49, 2415-2421.

Varga, Z., Wacha, A., Vainio, U., Gummel, J. \& Bóta, A. (2012). Chem. Phys. Lipids, 165, 387-392.

Woodle, M. C. (1995). Adv. Drug Deliv. Rev. 16, 249-265. 